\title{
Separation of paramagnetic and ferrimagnetic susceptibilities using low temperature magnetic susceptibilities and comparison with high field methods
}

\author{
Carl Richter *, Ben A. van der Pluijm \\ Department of Geological Sciences, University of Michigan, Ann Arbor, Michigan 48109, USA \\ (Received 13 November 1992; accepted 3 September 1993)
}

\begin{abstract}
Magnetic susceptibility of rocks can be dominated by diamagnetic and paramagnetic matrix minerals, ferrimagnetic and antiferromagnetic trace minerals, or a combination. The interpretation of magnetic fabric data (anisotropy of magnetic susceptibility, AMS) hinges on the qualitative and quantitative analysis of the sources of magnetic susceptibility. We discuss two methods that quantify the contribution of the different groups to the AMS: (1) comparative measurements of the magnetic susceptibility in low fields and high fields and (2) heating curves from $77 \mathrm{~K}$ to room temperature (low temperature magnetic susceptibility, LTMS). Method 1 measures paramagnetic, diamagnetic, and antiferromagnetic susceptibilities above the saturation magnetization of the ferrimagnetic minerals and method 2 interprets heating curves based on the fact that only the paramagnetic susceptibility is a function of temperature (Curie-Weiss law). Curie constants, paramagnetic Curie temperatures, and phase transitions (Verwey at $118 \mathrm{~K}$ : magnetite; Morin at $263 \mathrm{~K}$ : hematite) are diagnostic for specific minerals and provide further information about the contributing minerals of the sample. The relative contribution of the ferrimagnetic and paramagnetic minerals to the total susceptibility can be estimated from both methods with the same precision, if antiferromagnetic and diamagnetic contributions are insignificant. However, the LTMS method requires only simple equipment and procedures. The low temperature method can be extended to the three-dimensional case to decompose the total susceptibility tensor into its paramagnetic and ferrimagnetic sub-tensors (low temperature AMS, LTAMS). LTMS and LTAMS are powerful additions to the group of magnetic fabric methods that allow the quantification of mineral preferred orientation in natural samples.
\end{abstract}

\section{Introduction}

After its introduction to the geosciences (Ising, 1942; Graham, 1966), measurements of the anisotropy of magnetic susceptibility (AMS; e.g. reviews by Hrouda, 1982; Borradaile, 1988) have been used to obtain preferred mineral orientation data in sedimentology, volcanology, and structural geology. The AMS ellipsoid has been proposed to correlate with the finite strain ellipsoid

\footnotetext{
* Corresponding author at: Ocean Drilling Program and Department of Geology and Geophysics, Texas A\&M University, 1000 Discovery Drive, College Station, TX 77845, USA
}

and relationships between the two tensors were determined empirically (review by Borradaile, 1991), in experiments (e.g. Borradaile and $\mathrm{Pu}$ umala, 1989), and mathematically (Owens, 1974; Hrouda, 1980; Richter, 1992). Strong influences of the rock composition on the AMS ellipsoid (Owens and Bamford, 1976; Borradaile, 1987) and the fact that more frequently paramagnetic minerals and not, as it was previously thought, the ferrimagnetic trace minerals dominate the AMS of natural rocks (Rochette, 1987; Housen and van der Pluijm, 1990) led to a re-evaluation of the value of the method. It was concluded that AMS measurements are not interpretable if the sources of magnetic susceptibility are unknown. 
AMS measures the degree of preferred orientation of the short and long axes of the magnetites in a ferrimagnetic-dominated rock and the crystallographic preferred orientation of mica basal planes in a mica-dominated rock (Uyeda et al., 1963). In many cases both groups contribute significantly to the AMS and an interpretation of the data is only possible if the ferrimagnetic or the paramagnetic part can be separated from the total AMS tensor. Under these conditions, AMS is a powerful petrofabric tool, similar to a U-stage or an X-ray texture goniometer if the sources of magnetic susceptibility are known. Moreover, if strain and mineral preferred orientation relationships correlate well (March, 1932; Oertel, 1983; Richter et al., 1993), AMS provides a direct measurement of finite strain.

The use of AMS data as a method to determine mineral preferred orientations requires the identification of the sources of magnetic susceptibility and if more than one mineral contributes to the magnetic susceptibility the quantification and separation of the different sub-tensors (ferrimagnetic, paramagnetic, and diamagnetic subtensors). Four separation methods have been proposed to analyze bulk properties quantitatively: (1) a numerical method that uses data reduction (Henry and Daly, 1983; Henry, 1985), (2) the anisotropy of remanence (e.g. Jackson, 1991), (3) heating curves of the susceptibility from low temperatures (typically $77 \mathrm{~K}$ ) to room temperature (Schultz-Krutisch and Heller, 1985; Jover et al., 1989), (4) the comparison between high field and low field susceptibilities (Rochette and Fillion, 1988; Hrouda and Jelinek, 1990). Method 1 (Henry method) does not work on the specimen scale and is only valid under the assumption that variations in the magnetic susceptibility of the site result from variations in the amount of ferrimagnetic minerals. The second method, the anisotropy of remanence (anisotropy of anhysteretic remanence or isothermal remanence) that can be acquired only by ferrimagnetic minerals, yields results that are not simply comparable with those obtained from any other method. The reason is that the acquisition of a remanent magnetization is a function of physical parameters such as grain size, coercitive force, and applied field
(Stephenson et al., 1986; Jackson et al., 1988; Jackson, 1991). Methods 3 and 4 are based on the specific behavior of the magnetic susceptibility in different fields and temperatures and yield a unique solution. Methods to resolve both high field and low field anisotropy have been developed, but require specific rock magnetic equipment that is not available to most laboratories. Rochette and Fillion (1988), for example, use a rotating sample in a cryogenic magnetometer at fields from 0 to $4 \mathrm{~T}$ and Parma (1988) and Hrouda and Jelinek (1990) use a high field torque meter.

In this paper we discuss a low temperature method for bulk properties (experimental procedures and data analysis) and further expand its application. Throughout we will refer to this method as low temperature magnetic susceptibility (LTMS), which needs only simple equipment (thermocouple and liquid nitrogen) in addition to a susceptibility meter. The LTMS will be compared with results obtained from the high field/ low field method on a series of natural samples from different studies. Further on, we will extend the low temperature method to the three dimensional case to demonstrate how to separate the ferrimagnetic from the paramagnetic part of the total AMS tensor, i.e. how to obtain the paramagnetic, and the ferrimagnetic sub-tensors. We will refer to this method as low temperature anisotropy of magnetic susceptibility (LTAMS).

The aim of our paper is to provide the basic theory and technology for the identification and separation of ferrimagnetic and paramagnetic fabrics, which is essential for any interpretation of magnetic susceptibility data with standard AMS equipment.

\section{Identification of the sources of magnetic sus- ceptibility}

\subsection{Low field and high field susceptibilities}

The magnetic susceptibility $(k)$ is the dimensionless proportionality factor between the magnitude of induced magnetization (M) and the applied magnetic field strength $(H): M_{\mathrm{i}}=k_{\mathrm{ij}} H_{\mathrm{j}}$ (e.g. Hrouda, 1982; Nye, 1985), where $k_{\mathrm{ij}}$ is a 
second-rank symmetric tensor. This relationship , is valid for low fields and high saturating fields.

The low field susceptibility $\left(k_{0}\right)$ is usually determined at fields in the order of $0.1-0.5 \mathrm{mT}$ and measures the induced magnetization of all minerals (diamagnetic, paramagnetic, antiferromagnetic, and ferrimagnetic) in the rock under investigation

$k_{0}=k_{\text {para }}+k_{\text {dia }}+k_{\text {antiferro }}+k_{\text {ferri }}$

The applied field is so weak that it has no effect on the remanent magnetization of the sample.

Figure 1(a) shows the induced magnetization of a sample as a function of an applied field (hysteresis loop) with up to $1 \mathrm{~T}$ field strength (high field). In this example the ferrimagnetic minerals have reached their saturation magnetization $M_{\text {sat }}$ at c. $0.2 \mathrm{~T}$. At fields above $M_{\text {sat }}$ only the paramagnetic, diamagnetic, and antiferromagnetic minerals (matrix minerals) respond to the external field. The induced magnetization in fields above $M_{\text {sat }}$ can be used to calculate the high field susceptibility

$\tan (\alpha)=k_{\mathrm{HF}}=k_{\text {para }}+k_{\mathrm{dia}}+k_{\text {antiferro }}$

where $\alpha$ is the slope of the regression line (Fig. 1(a)). The difference between the low field and the high field susceptibility of a sample yields the ferrimagnetic susceptibility

$k_{\text {ferri }}=k_{0}-k_{\mathrm{HF}}$

A correction for the diamagnetic minerals (not the total diamagnetic susceptibility), like quartz, feldspar, or calcite is possible if their volume $V_{\text {dia }}$ is estimated and a constant value of $-14 \times 10^{-6}$ (e.g. Nye, 1985; Hrouda, 1986; Borradaile, 1987) is used as the diamagnetic volume susceptibility. The total diamagnetic susceptibility, however, also contains the diamagnetic contribution of all other minerals (paramagnetic and ferrimagnetic), which is in the order of $-1 \times 10^{-5}$ for most rockforming minerals.

Figure 1(b) shows an example of the magnetization behavior of a paramagnetic schist in fields up to $1 \mathrm{~T}$. The straight line demonstrates (1) that a ferrimagnetic remanence is absent and (2) that the susceptibility is the same in low and high fields $\left(k_{\mathrm{HF}}=k_{0} ; k_{0}=1.91 \times 10^{-4}\right.$ for this exam-
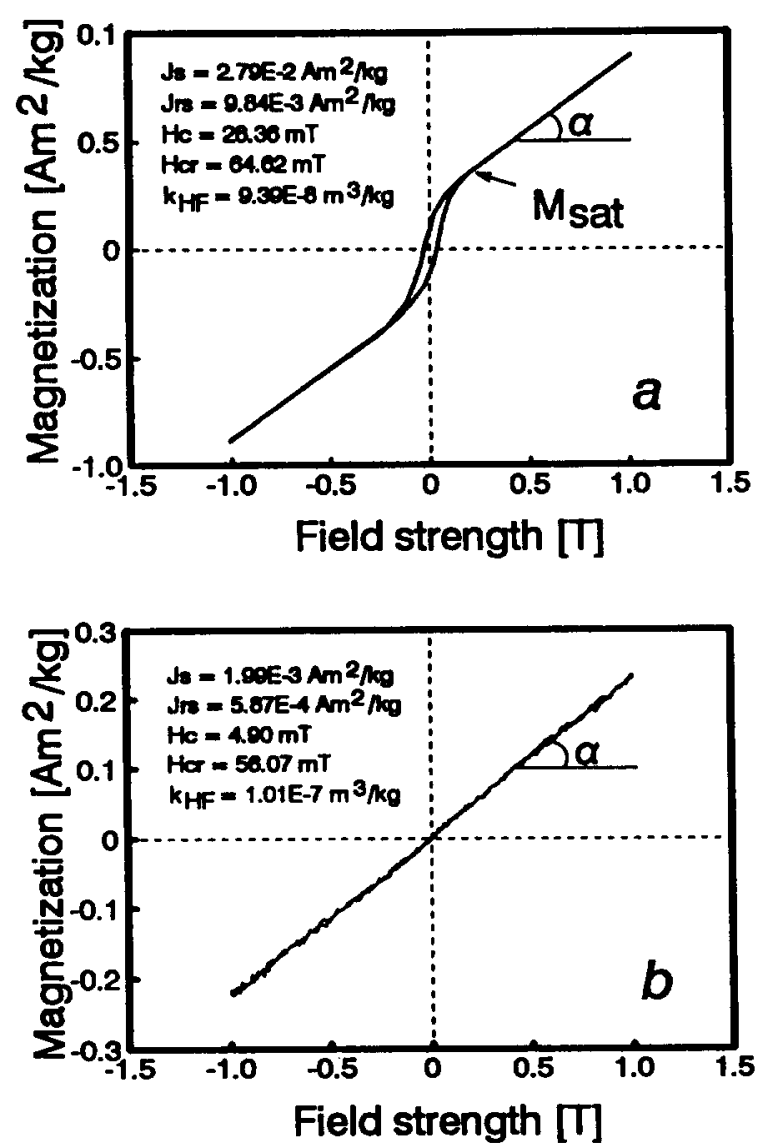

Fig. 1. High field susceptibility is determined in fields above the saturation magnetization $\left(M_{\mathrm{sat}}\right)$ of ferrimagnetic minerals ( $k_{\mathrm{HF}}=M / H$; mass susceptibility in SI units). (a) The hysteresis loop of a magnetite bearing sample; the ferrimagnetic minerals are saturated at $0.2 \mathrm{~T}$. In higher fields only paramagnetic, diamagnetic, and antiferromagnetic minerals respond to the applied field and their susceptibility can be determined from the slope $\alpha$ of the linear part of the loop. (b) The hysteresis loop is a straight line if ferrimagnetic minerals are absent or negligible; the low field and the high field measurements are identical and both measure the susceptibility of the matrix minerals.

ple). Hence, there is virtually no contribution of a ferrimagnetic phase and the low field anisotropy (AMS) measures the crystallographic preferred orientation of the matrix minerals.

Low field measurements were carried out on a Sapphire Instruments SI-2 susceptibility meter (applied field: $0.1 \mathrm{mT}$ ) and high field measurements on a vibrating sample magnetometer (applied field up to $1.5 \mathrm{~T}$ ). 


\subsection{Analysis of low temperature magnetic suscepti- bility (LTMS)}

The low temperature method investigates the temperature dependence of the magnetic susceptibility. The paramagnetic susceptibility $\left(k_{\text {para }}\right)$ is a function of temperature $(T)$ and is described by the Curie-Weiss law

$k_{\text {para }}=C /(T-\theta)$

with $C$ the Curie constant and $\theta$ the paramagnetic Curie temperature. $\theta$ can vary from -20 to $40 \mathrm{~K}$ for biotite (Bouchez et al., 1990) and is negligible for muscovite and most chlorite bearing rocks. The Curie-Weiss law is valid only for paramagnetic materials. Antiferromagnetic, diamagnetic, and most ferrimagnetic minerals have a temperature independent susceptibility in the range used here (77-295 K). Analysis of susceptibility vs. temperature therefore allows us to identify whether the sample is entirely paramagnetic, diamagnetic, or ferrimagnetic, or contains a mixture of these groups. Two methods have been proposed to measure the temperature dependence of a sample: (1) heating up to ca. $800^{\circ} \mathrm{C}$ (e.g. Zapletal, 1990); and (2) cooling to low temperatures (Rochette and Vialon, 1984; SchultzKrutisch and Heller, 1985). Heating is destructive and alters the sample (oxidation, dehydration, and other irreversible mineral transitions) and requires a specially equipped furnace. Cooling, on the other hand, can be carried out by emerging the sample into a refrigerated gas (e.g. nitrogen at $77 \mathrm{~K}$ or helium at $4.2 \mathrm{~K}$ ) and does not alter the sample. This requires only slight equipment modification.

\subsection{Experimental procedures}

We use a SI-2 (Sapphire Instruments) susceptibility meter in combination with a thermocouple ( $1 \mathrm{~mm}$ probe diameter; accuracy $\pm 1^{\circ}$, type $\mathrm{K}$ ) connected to a hand-held digital thermometer for temperature control. The sample $(2.1 \times 2.4$ standard cylinder) has a $1 \mathrm{~mm}$ diameter hole for the thermocouple. It is not possible with this setup to measure the susceptibility-temperature $k(T)$ curve directly, because the thermocouple inter- feres with the susceptibility readings. Therefore, we first cool the sample down to $77 \mathrm{~K}$ (ca. $25 \mathrm{~min}$ fully emerged in a liquid nitrogen bath) and measure the temperature during heating to room temperature as a function of time. A computer utility automatically records the time at which a reading is entered and stores the temperaturetime $T(t)$ data. This step can be fully automated. The sample is then cooled again and the susceptibility is measured as a function of time $k(t)$. The $T(t)$ and $k(t)$ curves are combined to a $k(T)$ curve using a computer routine. The time intervals between the temperature and the susceptibility readings can be independently selected and the same $T(t)$ curve can be used for different specimens with the same lithology. Typical heating times are 15-30 min depending on sample size and rock composition. A better resolution and precision can be obtained by slowing down the heating process with a styrofoam insulated sample holder. Depending on the insulation the heating process takes 2-3 times longer, which allows the determination of extremely well defined curves and accurate detection of phase transitions (see below).

The temperature distribution inside a cylindrical specimen without insulation shows a gradient with lowest temperatures in the core (Fig. 2). Initially, the surface heats up fastest, but after about 3 min surface effects from condensing liquids keep the temperatures at the rim down. The temperature distribution is homogenous after about $20 \mathrm{~min}$. The best place to measure the average temperature of such a specimen is approximately half-way between core and rim. Measurements from the center of the specimen can have an up to $8 \mathrm{~K}$ lower reading than the average temperature, which, because we are dealing with the bulk magnetic properties, introduces a slight error. The temperature of insulated specimens has more time to equilibrate, and hence has a smaller gradient from the core to the rim.

\subsection{LTMS results from paramagnetic and ferri- magnetic samples}

The LTMS data are represented in diagrams that plot the normalized reciprocal $\left(k_{0} / k\right)$ or the 
reciprocal magnetic susceptibility $(1 / k)$ vs. temperature. Both functions transform a paramagnetic curve into a straight line. The normalized plot is preferable for representation and the $1 / k$ curve shows differences in absolute values that can be used to determine $C$ and $\theta$. Figure 3(a) shows the $k_{0} / k$ vs. $T$ curve of a muscovite-chlorite bearing schist. The regression line goes through the origin of the graph and $R^{2}$ is very close to 1 . Hence, the sample obeys the CurieWeiss law perfectly. The paramagnetic Curie temperature $\theta$ is zero and ferrimagnetic minerals do not contribute to the susceptibility. AMS data from this specimen can be used to quantify the crystallographic preferred orientation of the paramagnetic minerals (muscovite and chlorite) present. Figure 3(b) shows the low temperature behavior of a magnetite separate. The susceptibility jump around $118 \mathrm{~K}$ is caused by a lattice transition (orthorhombic to cubic (Verwey) transition), which is a diagnostic criterion for the presences of magnetite. The susceptibility above $118 \mathrm{~K}$ shows no temperature dependence and

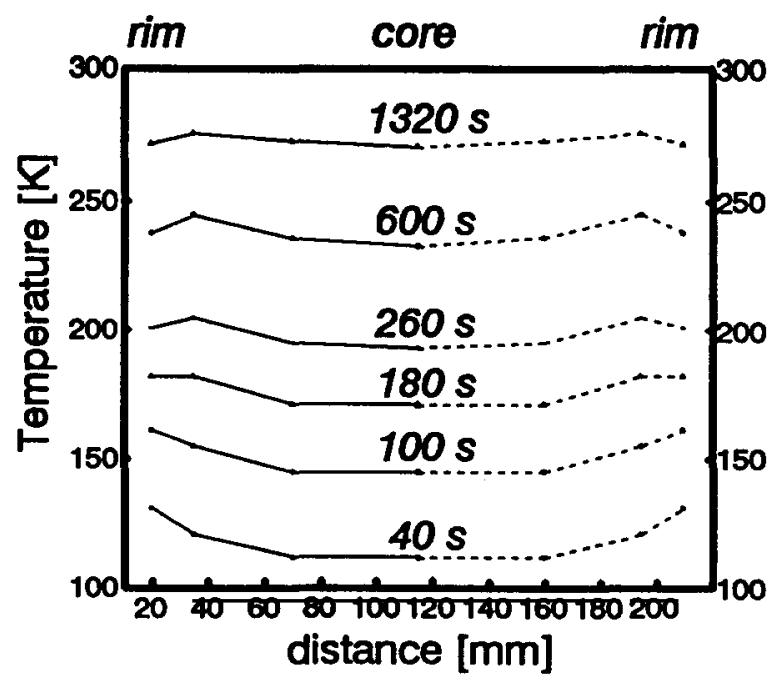

Fig. 2. Temperature profile through a standard-sized cylinder (diameter: $2.4 \mathrm{~cm}$; length: $2.2 \mathrm{~cm}$ ) at different time intervals during heating from $77 \mathrm{~K}$ to room temperature. After $180 \mathrm{~s}$ the condensation of water insulates the sample and decreases the temperature gradient. Dashed lines are interpolations assuming symmetry.
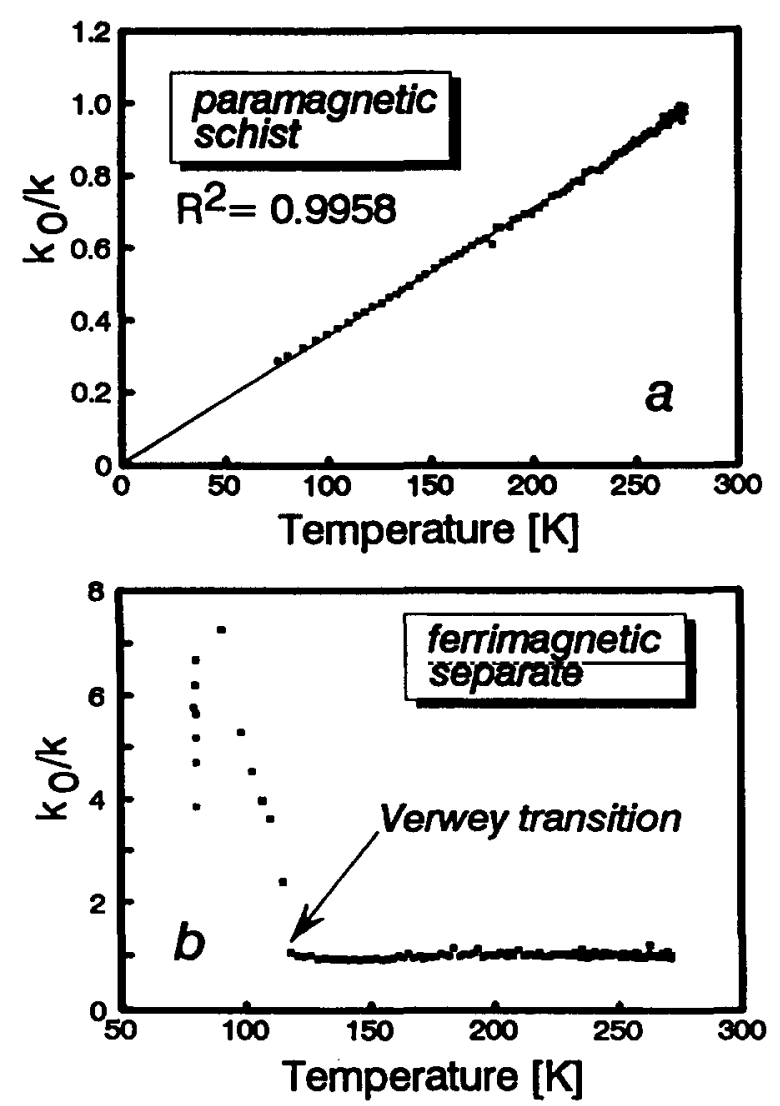

Fig. 3. The normalized reciprocal of the magnetic susceptibility is plotted as a function of temperature. (a) The $k_{0} / k$ curve of a mica-dominated schist is a straight line with a regression coefficient $R^{2}$ close to 1 and an intercept at zero. The magnetic susceptibility obeys the Curie-Weiss law perfectly; the paramagnetic Curie temperature is zero, ferrimagnetic influences are not detectable. (b) The $k_{0} / k$ curve of separated magnetite minerals shows the lattice transition at $118 \mathrm{~K}$ (Verwey transition) and no temperature dependence between $120 \mathrm{~K}$ and $273 \mathrm{~K}$

remains constant until room temperature is reached. Magnetite-dominated rock samples show the same behavior, and AMS in this case measures the shape anisotropy of magnetite. The temperature dependence of the susceptibility of titanomagnetites and superparamagnetic magnetites is, however, not negligible, which excludes these minerals from a simple low temperature analysis. 


\subsection{Mixed ferrimagnetic and paramagnetic behav- ior}

In many samples paramagnetic and ferrimagnetic minerals both contribute to the susceptibility, and heating curves are the result of the superposition of the paramagnetic and ferrimagnetic effects. Figure 4 shows the results of a model calculation that uses ideal paramagnetic $(\theta=0)$ and ferrimagnetic $(k(T)=$ constant) compounds. The paramagnetic and ferrimagnetic values at each temperature step were added to obtain the superimposed curve. The ferrimagnetic susceptibility of the initial mixture is $90 \%$ of the total susceptibility. From the initial mixture an assumed ferrimagnetic susceptibility $k_{\text {ferri }}$ (given in percent of the total ferrimagnetic susceptibility in Fig. 4) was removed, and the $\left(k_{0}-k_{\text {ferri }}\right) /(k-$ $k_{\text {ferri }}$ ) curve plotted vs. temperature. The resulting curves have a convex shape that evolves to a straight line (regression coefficient $R^{2}$ reaches a maximum), when the ferrimagnetic contribution is entirely removed. In a natural sample $k_{\text {ferri }}$ actually represents the sum of $k_{\text {ferri }}+k_{\mathrm{dia}}+$ $k_{\text {antiferro }}$, depending on the rock composition.

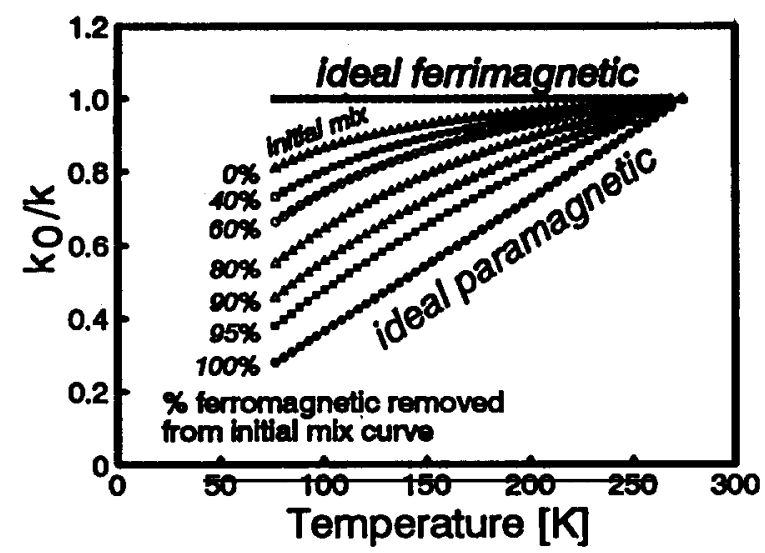

Fig. 4. Numerical model of ideal paramagnetic and ferrimagnetic curves in a $k_{0} / \mathrm{k}$ vs. $T$ plot. Both curves were added to an initial mixture that has a ratio of $90: 10$ ferrimagnetic to paramagnetic contribution to the total susceptibility. The ferrimagnetic contribution was stepwise removed from the initial curve; numbers in [\%] are removed percent of ferrimagnetic contribution. The resulting curves have a convex shape that evolves into a straight line, as the ferrimagnetic contribution is removed.
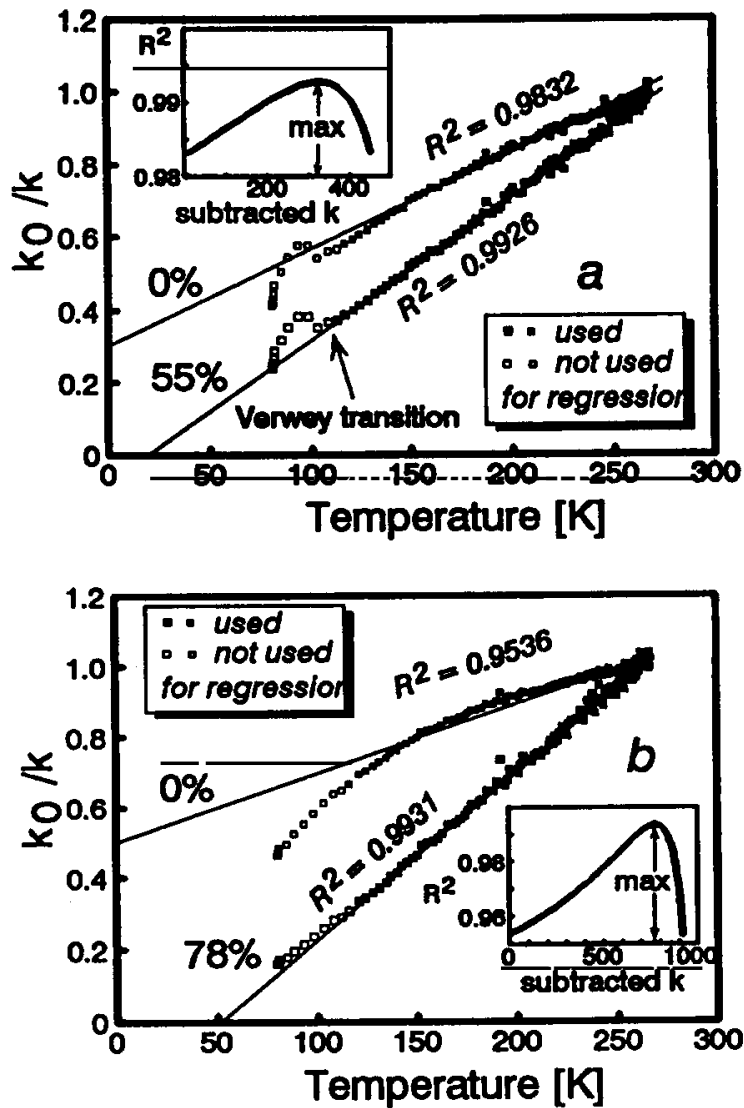

Fig. 5. Magnetic susceptibility $\left(k_{0} / k\right)$ as a function of temperature of paramagnetic and ferrimagnetic mixtures. Regression lines are calculated for $T$ greater than $120 \mathrm{~K}$ to exclude effects from the Verwey transition and from deviations from the Curie-Weiss law that occur at low temperatures. (a) Artificial sample: the upper curve is the heating curve with $0 \%$ ferrimagnetic susceptibility subtracted, the lower curve is a straight line representing the paramagnetic contribution to the initial curve. Inset shows the maximization of the regression coefficient $R^{2}$ to determine the paramagnetic part of the curve and the temperature independent part of the susceptibility $\left(k_{\text {ferri }}+k_{\text {dia }}+k_{\text {antiferro }}\right)$; subtracted susceptibility $k$ in SI volume units $\left(\times 10^{-6}\right)$. The temperature independent susceptibility is $55 \%$ of the total susceptibility. (b) Natural sample with $78 \%$ ferrimagnetic contribution and no visible Verwey transition. Compare the shape of the curves with the theoretical curves (Fig. 4).

Figures 5(a) and 5(b) show the low temperature analysis of two samples consisting of a paramagnetic and a ferrimagnetic part. The paramagnetic susceptibility is determined by subtracting 
an assumed ferrimagnetic susceptibility $k_{\text {ferri }}$ from each data point; $k_{\text {ferri }}$ is continuously increased, until the regression coefficient $R^{2}$ reaches a maximum. The insets of Figs. 5(a) and 5(b) show how $R^{2}$ increases as the convex curves are transformed into a straight entirely paramagnetic line and rapidly decreases as the curves become concave. The best fit line is defined as

$$
k^{-1}(T)=T / C-\theta / C
$$
line

Comparison with the general equation for a

$$
y=m x+b
$$

where $(m)$ is the slope and $(b)$ the intercept, rapidly yields the Curie constant $C\left(C=m^{-1}\right)$ and the paramagnetic Curie temperature $\theta(\theta=$ $-b / m)$ of the sample. The sample in Fig. 5(a) is an artificial biotite-magnetite mixture and shows the Verwey transition which is excluded from our regression analysis. The negative intercept of the best-fit line yields a paramagnetic Curie temperature of $\theta=19.6 \mathrm{~K}$. Figure 5(b) shows the heating curve of a natural magnetite-bearing schist without a visible Verwey transition. The ferrimagnetic susceptibility in this example is $78 \%$ of the room temperature susceptibility. The convex shape of the initial curve is straightened out as $R^{2}$ increases from 0.9536 to its maximum value at 0.9931 . We have developed a computer program that iteratively straightens out the heating curve until $R^{2}$ reaches a maximum and calculates $C$ and $\theta$ from the best-fit regression line.

We caution that a quantification of the contribution to the magnetic susceptibility of the various minerals with the low temperature method can only be obtained for multidomain magnetite, not for titanomagnetites or superparamagnetic magnetites because their susceptibility is not temperature independent. Hematite bearing rocks can only be qualitatively analyzed. The important assumption that the temperature dependence of the ferrimagnetic susceptibility is negligible can be examined on a magnetic separate (e.g. Fig. 3(b)). If the temperature dependence of the magnetite susceptibility is not negligible, $k_{\text {ferri }}$ must be determined as a function of temperature $k_{\text {ferri }}(T)$ and accounted for in the iteration proce- dure. A bulk estimate for the diamagnetic susceptibility if the volume of diamagnetic minerals $\left(k=-14 \times 10^{-6}\right)$ is known and a mean value of $k=-1 \times 10^{-5}$ for the diamagnetic contribution of the paramagnetic minerals is used can be subtracted from the ferrimagnetic susceptibility.

\subsection{Comparison of LTMS and high field / low field methods}

The high field/low field method and the LTMS method both quantify the sources of magnetic susceptibility. The fundamental difference is that the high field method separates $\left(k_{\mathrm{dia}}+\right.$ $k_{\text {para }}+k_{\text {antiferro }}$ ) from $k_{\text {ferri }}$ and that the LTMS method separates $\left(k_{\text {ferri }}+k_{\text {dia }}+k_{\text {antiferro }}\right)$ from $k_{\text {para }}$. Thus the results from both methods are only comparable if $k_{\text {dia }}$ and $k_{\text {antiferro }}$ are negligible, i.e. in rocks that are not dominated by diamagnetic minerals (quartz, feldspar, or calcite) or antiferromagnetic minerals (hematite or goethite). Only the combination of both methods yields unique solutions for $k_{\text {para }}, k_{\text {ferri }}$, and $k_{\text {dia }}+$ $k_{\text {antiferro }}$ in the general case. However, in practice the $k_{\mathrm{dia}}$ and $k_{\text {antiferro }}$ are often negligible, or $k_{\text {dia }}$ can be estimated. We have compared results ob-

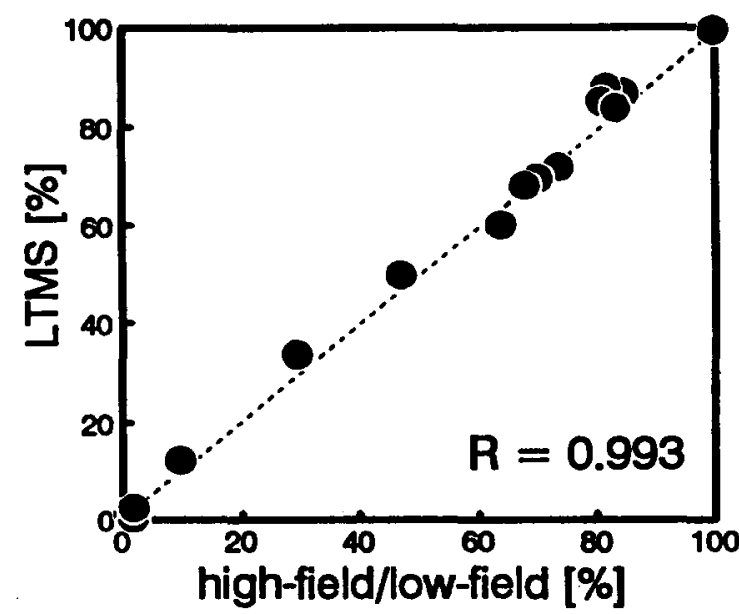

Fig. 6. Comparison of the ferrimagnetic susceptibility determined independently from the low temperature method and from the high field-low field method. Both methods yield the same estimate because of negligible influences from diamagnetic and antiferromagnetic minerals; regression coefficient $R^{2}=0.993$. 
tained from both methods (Fig. 6) using a variety of samples from different localities (slates, phyllites, and mica schists from the Central and Southern Alps and mudstones from ODP hole $808 \mathrm{C}$ ) that have negligible diamagnetic and antiferromagnetic contributions. The graph (Fig. 6) shows a one-to-one correlation with a regression coefficient close to $1\left(R^{2}=0.993\right)$. The slope of the regression line is close to $1(0.977)$ and the intercept is close to zero $(0.1)$. The slight deviation between the two methods may arise from: (1) the physical properties were not determined strictly in the same direction, (2) not always the same specimens from one sample were used, (3) high field and low field-field susceptibilities were measured on different instruments, (4) the low temperature method assumes that the ferrimagnetic minerals have no temperature dependence and (5) diamagnetic and antiferromagnetic contributions were neglected. The sensitivity and drift of the equipment is not a significant quality factor, because the high field method takes about 300 readings during the hysteresis loop and the low temperature method, depending on the temperature interval used, is based on 100-140 measurements, which balances out most nonsystematic instrumental errors. The comparison shows that the correlation between the two methods is very good and that the low temperature method is equally effective in separating ferrimagnetic and paramagnetic components.

\section{Decomposing the magnetic susceptibility ten- sor}

Quantification of the sources of magnetic susceptibility provides an estimate of the contribution of the paramagnetic (LTMS) and ferrimagnetic (high field) minerals to the bulk magnetic susceptibility. This is the fundamental basis for the interpretation of samples that have been identified as dominated by ferrimagnetic or paramagnetic minerals. If both groups contribute significantly, a separation of the paramagnetic and the ferrimagnetic part of the magnetic susceptibility tensor is necessary for a quantitative interpretation. Low temperature AMS (LTAMS) de- termines the susceptibility tensor at $77 \mathrm{~K}$ (e.g. Ihmlé et al., 1989). This approach is not a true separation method and only uses the fact that the paramagnetic bulk susceptibility is strongly enhanced at low temperatures. The Curie-Weiss law (assuming that $\theta=0$ ) shows that the paramagnetic susceptibility will be enhanced by a factor of factor 3.8 if the temperature is lowered from 290 to $77 \mathrm{~K}$

$$
(C / 77) /(C / 290)=3.8
$$

However, the LTAMS of a paramagnetic mineral is not necessarily the same as the AMS at
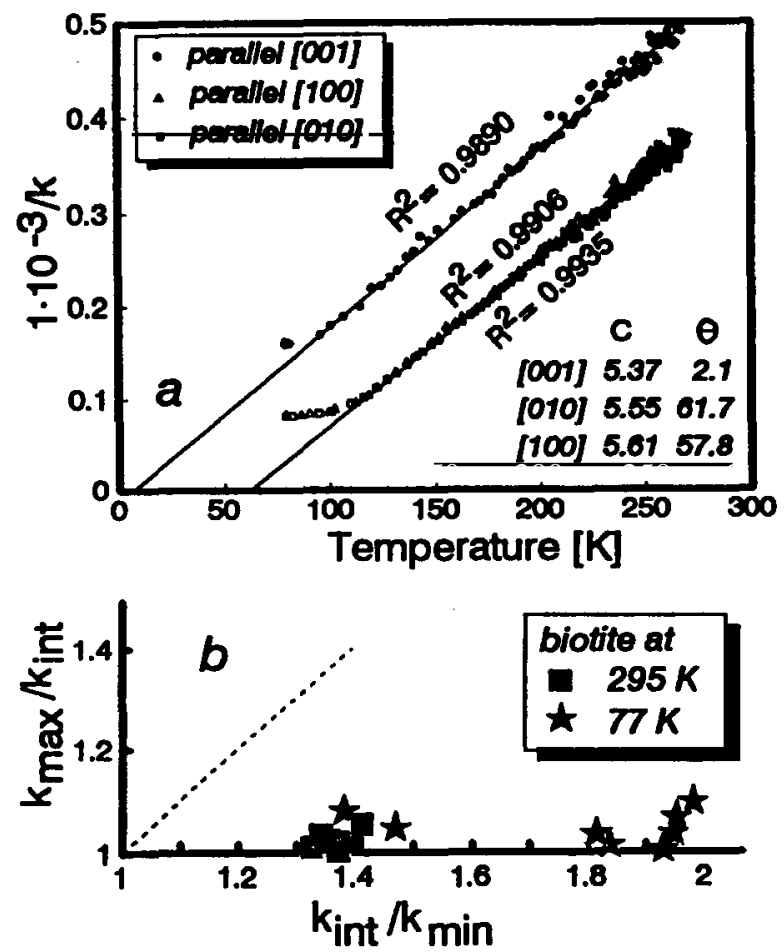

Fig. 7. (a) Low temperature susceptibility curves in different crystallographic orientations of a single biotite mineral. Properties parallel [100] and [010] overlap; the paramagnetic Curie temperature $\theta$ differs significantly between the basal plane and crystallographic $c$ axis. Deviation from the Curie-Weiss behavior occurs at temperatures below $110 \mathrm{~K}$. Values for the Curie constant are $\times 10^{-2}$. (b) Comparison of the room temperature AMS and the LTAMS of single biotite minerals. Caused by the anisotropy of $\theta$ and deviation from the CurieWeiss law at low temperatures, AMS is not temperature independent. The $77 \mathrm{~K}$ measurements have an enhanced anisotropy and an up to 4 times higher bulk susceptibility. 
room temperature. The Curie-Weiss law demonstrates that the paramagnetic anisotropy is temperature independent only if $\theta$ is not a function of the crystallographic orientation. Our LTMS curves of a single biotite crystal (Fig. 7(a)) parallel to the crystallographic axes ([001], [010], [100]) show that $C$ and $\theta$ are directional dependent. Within the biotite basal plane the values are almost identical $(C=5.55-5.61 \mathrm{~K} ; \theta=58-62 \mathrm{~K})$ but differ drastically from $c$ axis parallel data $(C=5.37 \mathrm{~K} ; \theta=2.1 \mathrm{~K})$. Ferrimagnetic inclusions, which are likely to occur in biotite crystals, appear to have no effect on the magnetic susceptibility of this crystal. At temperatures below $110 \mathrm{~K}$ the measurements no longer obey the CurieWeiss law (Fig. 7(a)), which is probably a result of ordering effects caused by the reduced atomic motion close to the paramagnetic Curie temperature $(57-62 \mathrm{~K})$ or by superparamagnetic behavior. The magnetic anisotropy of single biotite minerals measured at room temperature and at $77 \mathrm{~K}$ is shown in Fig. 7(b) in a Flinn-type diagram. The change in the degree of anisotropy $\left(k_{\max } / k_{\min }\right.$, with $k_{\max } \geqslant k_{\text {int }} \geqslant k_{\min }$ as the principal susceptibilities) is a result of the directional dependence of $\theta$. The $k_{\max } / k_{\text {int }}$ ratio ( $C$ and $\theta$ for [100] and [010] are almost identical) stays almost constant with decreasing temperatures but
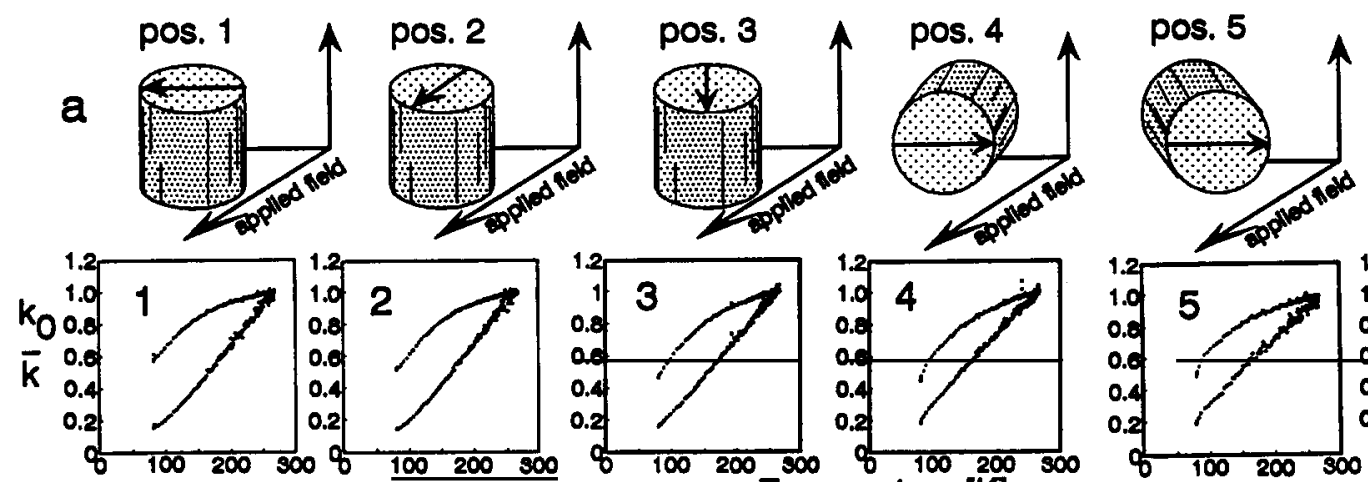

pos. 6

b
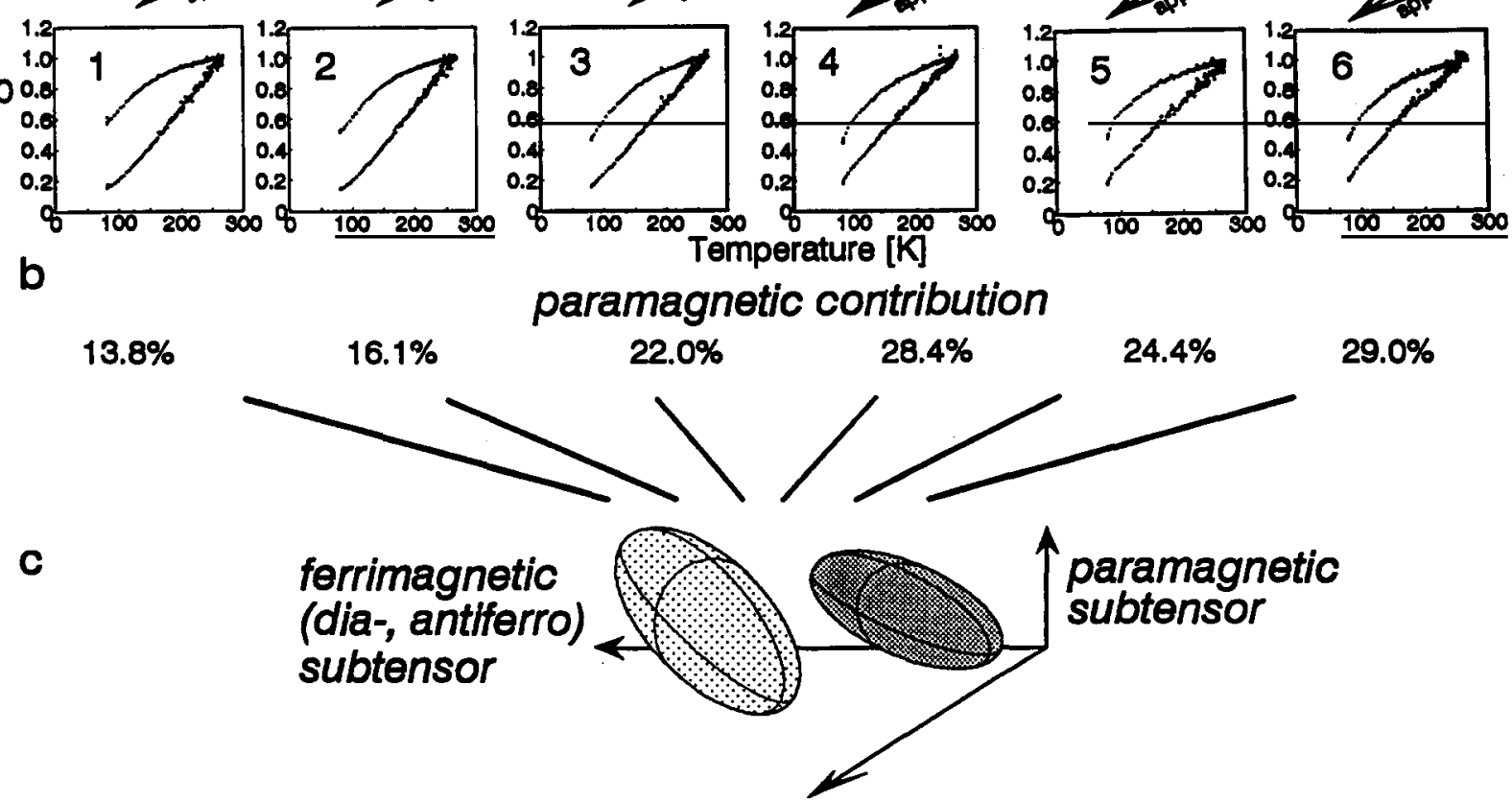

Fig. 8. Principle of the separation of the ferrimagnetic and paramagnetic sub-tensors from the total AMS tensor using LTMS curves. (a) Six specimen orientations are used to define the susceptibility tensor. Arrows define coordinate system and direction of applied field. (b) The relative amount of paramagnetic and ferrimagnetic susceptibilities is determined in six orientations from heating curves. The upper curve is the original heating curve and the lower curve the paramagnetic remnant. (c) The ferrimagnetic susceptibilities are combined to the ferrimagnetic sub-tensor and the six paramagnetic susceptibilities to the paramagnetic sub-tensor, which yield the magnitudes and principal directions of the ferrimagnetic and the paramagnetic susceptibility ellipsoid. 
the $k_{\text {int }} / k_{\min }$ ratio ( $\theta$ is $2 \mathrm{~K}$ parallel [001] and ca. $60 \mathrm{~K}$ in the other directions) increases from ca. 1.4 to ca. 1.9 (Fig. 7(b)). Two of the single biotite minerals have a $\theta$ close to zero and do not change the AMS geometry at different temperatures. The principal directions, on the other hand, remain the same and because of the larger susceptibility differences they are better defined at low temperatures. In conclusion, low temperature anisotropies can only be compared with room temperature anisotropies if $\theta$ is directionally independent. This seems to be the case for the muscovite bearing rocks we have measured so far, but not if biotite is present, in which case a correction is required.

To obtain a true separation of the paramagnetic part of the total susceptibility tensor we propose a quantitative analysis of several heating curves from different orientations of the same specimen (Fig. 8). The percentage of the paramagnetic contribution and the volume susceptibility are determined in six orientations of the specimen from LTMS curves (Fig. 8(a) and (b)). The results yield the paramagnetic susceptibilities in six directions, which are combined to derive the paramagnetic sub-tensor of the complete AMS tensor (Fig. 8(c)). Our example is a natural sample, where the ferrimagnetic and paramagnetic contributions were determined in six sample positions to define the paramagnetic and ferrimagnetic subtensors. Consequently, the ferrimagnetic subtensor can be obtained from this analysis in the case that no antiferromagnetic minerals contribute and the weak diamagnetic and isotropic contribution is neglected. Currently, we are testing this method in a group of artificial and natural samples.

\section{Availability of computer programs}

Measuring and analysis programs for DOS computers (executable version and Turbo-Pascal 6.0 source code) can be obtained by sending a formatted disc in a self addressed mailer to $\mathrm{C}$. Richter.

\section{Acknowledgments}

Research was supported by the Deutsche Forschungsgemeinschaft, grant RI576/1-2, the National Science Foundation, grant EAR9119196, and the Scott Turner Fund of the University of Michigan. We are especially indebted to Friedrich Heller (Zürich) for detailed information on his 1985 paper, to Mike Stupavsky (Sapphire Instruments) for modifications to our equipment, and to Bernie Housen and John Stamatakos for discussion. Don Cederquist is thanked for patiently carrying out several low temperature experiments. Mike Jackson (Minneapolis) and David Dunlop (Toronto) carried out initial high field measurements on some samples. High field and single mineral AMS measurements were performed at the Institute for Rock Magnetism (Minneapolis) with the support of Chris Hunt and Jim Marvin. This paper benefited considerably from insightful reviews by Pierre Rochette and an anonymous reviewer.

\section{References}

Borradaile, G.J., 1987. Anisotropy of magnetic susceptibility: rock composition versus strain. Tectonophysics, 138: 327 329.

Borradaile, G.J., 1988. Magnetic susceptibility, petrofabrics and strain-a review. Tectonophysics, 156: 1-20.

Borradaile, G.J., 1991. Correlation of strain with anisotropy of magnetic susceptibility (AMS). Pure Appl. Geophys., 135: $15-29$.

Borradaile, G.J. and Puumala, M.A., 1989. Synthetic magnetic fabrics in a plasticene medium. Tectonophysics, 164: 73-78.

Bouchez, J.L., Gleizes, G., Djouadi, T. and Rochette, P., 1990. Microstructure and magnetic susceptibility applied to emplacement kinematics of granites: the example of the Foix pluton (French Pyrenees). Tectonophysics, 184, 157171.

Graham, J.W., 1966. Significance of magnetic anisotropy in Appalachian sedimentary rocks. In: J.S. Steinhart and T.J. Smith (Editors), The Earth Beneath the Continents. Geophys. Monogr., 10: 627-648.

Henry, B., 1985. Magnetic fabrics and superimposed deformations: example of Dalradian rocks from the southwest Highlands of Scotland. Phys. Earth Planet. Inter., 40: 187-200.

Henry, B. and Daly, L., 1983. From qualitative to quantitative magnetic anisotropy analysis: the prospect of finite strain calibration. Tectonophysics, 98: 327-336. 
Housen, B.A. and Van der Pluijm, B.A., 1990. Chlorite control of correlations between strain and anisotropy of magnetic susceptibility. Phys. Earth Planet. Inter., 61: 315-323.

Hrouda, F., 1980. Magnetocrystalline anisotropy of rocks and massive ores: a mathematical model study and its fabric implications. J. Struct. Geol., 2: 459-462.

Hrouda, F., 1982. Magnetic anisotropy of rocks and its application in geology and geophysics. Geophys. Surv., 5: 37-82.

Hrouda, F., 1986. The effect of quartz on the magnetic anisotropy of quartzite. Stud. Geophys. Geod., 30: 39-45.

Hrouda, F. and Jelinek, F., 1990. Resolution of ferrimagnetic and paramagnetic anisotropies in rocks, using combined low field and high field measurements. Geophys. J. Int., 103: 75-84.

Ihmlé, P.F., Hirt, A.M., Lowrie, W. and Dietrich, D., 1989. Inverse magnetic fabric in deformed limestones of the Morcles Nappe, Switzerland. Geophys. Res. Lett., 16: 1383-1386.

Ising, G., 1942. On the magnetic properties of varved clay. Ark. Mat. Astron. Fys., 29A: 1-37.

Jackson, M., 1991. Anisotropy of magnetic remanence: a brief review of mineralogical sources, physical origins, and geological applications, and comparison with susceptibility anisotropy. Pure Appl. Geophys., 136: 1-28.

Jackson, M., Gruber, W., Marvin, J. and Banerjee, S.K., 1988. Partial anhysteretic remanence and its anisotropy: applications and grain-size dependence. Geophys. Res. Lett., 15: 440-443.

Jover, O., Rochette, P., Lorand, J.P., Maeder, M. and Bouchez, J.L., 1989. Magnetic mineralogy of some granites from the French Massif Central: origin of their low field susceptibility. Phys. Earth Planet. Inter., 55: 79-92.

March, A., 1932. Mathematische Theorie der Regelung nach der Korngestalt bei affiner Deformation. Z. Kristallogr., 81: 285-297.

Nye, J.F., 1985. Physical Properties of Crystals. Oxford University Press, New York, 329 pp.

Oertel, G., 1983. The relationship of strain and preferred orientation of phyllosilicate grains in rocks-a review. Tectonophysics, 100: 413-447.
Owens, W.H., 1974. Mathematical model studies on factors affecting the magnetic anisotropy of deformed rocks. Tectonophysics, 24: 115-131.

Owens, W.H. and Bamford, D., 1976. Magnetic, seismic and other anisotropic properties of rock fabrics. Philos. Trans. R. Soc. London, Ser. A, 283: 55-68.

Parma, J., 1988. An automated torque meter for rapid measurement of high field magnetic anisotropy of rocks. Phys. Earth Planet. Inter., 51: 387-389.

Richter, C., 1992. Particle motion and the modelling of strain response in magnetic fabrics. Geophys. J. Int., 119: 451464.

Richter, C., Van der Pluijm, B.A. and Housen, B.A., 1993. The quantification of crystallographic preferred orientation using magnetic anisotropy. J. Struct. Geol., 15: 113116.

Rochette, P., 1987. Magnetic susceptibility of the rock matrix related to magnetic fabric studies. J. Struct. Geol., 9: 1015-1020.

Rochette, P. and Fillion, G., 1988. Identification of multicomponent anisotropies in rocks using various field and temperature values in a cryogenic magnetometer. Phys. Earth Planet. Inter., 51: 379-386.

Rochette, P. and Vialon, 1984. Development of planar and linear fabrics in Dauphinois shales and slates (French Alps) studied by magnetic anisotropy and its mineralogical control. J. Struct. Geol., 6: 33-88.

Schultz-Krutisch, T. and Heller, F., 1985. Measurement of magnetic susceptibility in Buntsandstein deposits from Southern Germany. J. Geophys., 57: 51-58.

Stephenson, A., Sadikun, N. and Potter, D.K., 1986. A theoretical and experimental comparison of the anisotropies of magnetic susceptibility and remanence in rocks and minerals. Geophys. J.R. Astron. Soc., 84: 185-200.

Uyeda, S., Fuller, M.D., Belshé, J.C. and Girdler, R.W., 1963 Anisotropy of magnetic susceptibility of rocks and minerals. J. Geophys. Res., 68: 279-291.

Zapletal, K., 1990. Low field susceptibility anisotropy of some biotite crystals. Phys. Earth Planet. Inter., 63: 85-97. 